\title{
Physically based computer graphics for realistic image formation to simulate optical measurement systems
}

\author{
Max-Gerd Retzlaff ${ }^{1,2}$, Johannes Hanika ${ }^{1}$, Jürgen Beyerer ${ }^{2,3}$, and Carsten Dachsbacher ${ }^{1}$ \\ ${ }^{1}$ Karlsruhe Institute of Technology (KIT), Institute for Visualization and Data Analysis (IVD), \\ Computer Graphics Group, Karlsruhe, Germany \\ ${ }^{2}$ Fraunhofer Institute of Optronics, System Technologies and Image Exploitation (IOSB), Karlsruhe, Germany \\ ${ }^{3}$ Karlsruhe Institute of Technology (KIT), Institute for Anthropomatics and Robotics, \\ Vision and Fusion Laboratory (IES), Karlsruhe, Germany \\ Correspondence to: Max-Gerd Retzlaff (retzlaff@kit.edu)
}

Received: 30 September 2016 - Revised: 21 January 2017 - Accepted: 28 February 2017 - Published: 8 May 2017

\begin{abstract}
Physically based image synthesis methods, a research direction in computer graphics (CG), are capable of simulating optical measuring systems in their entirety and thus constitute an interesting approach for the development, simulation, optimization, and validation of such systems. In addition, other CG methods, so-called procedural modeling techniques, can be used to quickly generate large sets of virtual samples and scenes thereof that comprise the same variety as physical testing objects and real scenes (e.g., if digitized sample data are not available or difficult to acquire). Appropriate image synthesis (rendering) techniques result in a realistic image formation for the virtual scenes, considering light sources, material, complex lens systems, and sensor properties, and can be used to evaluate and improve complex measuring systems and automated optical inspection (AOI) systems independent of a physical realization. In this paper, we provide an overview of suitable image synthesis methods and their characteristics, we discuss the challenges for the design and specification of a given measuring situation in order to allow for a reliable simulation and validation, and we describe an image generation pipeline suitable for the evaluation and optimization of measuring and AOI systems.
\end{abstract}

\section{Overview and state of the art in image synthesis}

Current physically based image synthesis techniques constitute a major leap compared to previously used, mostly phenomenological approaches. The simulation of light transport is at the core of physically based image synthesis methods and crucial to generate images that are on par with images made by physical image acquisition systems. Light transport simulation nowadays is almost exclusively computed using Monte Carlo (MC) or Markov chain Monte Carlo (MCMC) methods, which can account for complex light-matter interactions and naturally handle spectral emission, absorption, and scattering behavior (measured or derived from models) described by geometric optics. (MC)MC methods can also comprise the simulation of complex lens systems to accurately compute the resulting irradiance onto a virtual sensor.

Essentially, all (MC)MC rendering methods compute an estimate of the light transport by sampling, that is, stochasti- cally generating, paths on which light propagates from light sources to sensors, their main difference being the path sampling strategy. Until sufficient convergence, the variance of this estimation is apparent as noise in the images. Because of this, even simplistic realizations of these methods are versatile and, in principle, capable of achieving the desired, and required, results. However, their application is only practical when an (MC)MC method is used which is well suited for a given scenario; otherwise, the computation time can easily become prohibitively long, even in seemingly simple cases. For example, one would choose different methods for computing light transport for complex high-frequency light transport phenomena (recognizable by multiple glossy or specular reflection) than for highly scattering media.

As indicated, many different rendering algorithms and sampling strategies exist in the realm of (MC)MC methods, and they all exhibit different performance and noise charac- 




Figure 1. Illustration of basic ray tracing.

teristics, which are strongly linked to the type of light-matter interactions and the geometry configurations occurring in a scene. As such, it is not straightforward for the non-expert to select the appropriate method. For this purpose, we identify light interactions and phenomena constituting different challenges for the image synthesis, and discuss state-of-theart algorithms, such as, for example, path tracing and bidirectional path tracing (BDPT).

Particular attention has to be paid to the simulation of complex lens systems which can increase the computation time by orders of magnitude when implemented naively. We discuss the use of a state-of-the-art approach to efficient rendering with realistic lenses in the context of measuring and AOI systems.

\section{Image synthesis methods}

\subsection{Rendering, rasterization, and ray tracing}

Hughes et al. (2015) define the term rendering very concisely as referring to the process of integration of the light that arrives at each pixel of the image sensor inside a virtual camera in order to compute an image.

There are two major strategies for determining the color of an image pixel: rasterization and ray tracing.

Ray tracing, also sometimes referred to as ray casting, determines the visibility of surfaces by tracing rays of light from the virtual view point, that is, the viewer's eye or the image sensor, to the objects in the scene. The view point represents the center of origin and the image a window on an arbitrary view plane. For each pixel of the image a view ray is sent originating in the view point through the pixel into the scene in order to find an intersection with a surface. By recursive application of this ray casting, as illustrated in Fig. 1, one can compute complex light interactions and global illumination, that is, indirect illumination including, among others, reflections and shadows.



Figure 2. Illustration of the surface interaction at point $\boldsymbol{x}$.

Rasterization, on the other hand, projects geometric primitives one by one onto the image window. A depth buffer, also called a z-buffer, is utilized to determine the closest and thus visible primitive for each pixel.

Usually, the perspective projection is carried out in three steps. First, the projection transformation, expressed in homogeneous coordinates, is applied. Afterwards, the projective coordinates are dehomogenized by the normalization transformation, mapping the view frustum to the unit cube. The resulting device coordinates can then be mapped to image coordinates by discarding the depth component, as is done for orthographic projection.

All primitives can be processed in parallel using a single instruction multiple data (SIMD) approach and minor synchronization via the depth buffer. This allows for a very fast pipelined hardware implementation in the form of modern graphics processing units (GPUs).

Simplified, one could say that ray tracing starts with the pixels and then determines ray intersections with the scene geometry, while rasterization starts with the geometry, projecting it onto the image plane. The availability of modern GPUs makes rasterization feasible for interactive real-time application.

\subsection{Rendering equation and (Markov chain) Monte Carlo methods}

The surface interactions during ray tracing can be described easily, as illustrated in Fig. 2. As we start from the observer, or camera, and try to find paths to the light sources, ray tracing essentially considers the backward light transport. This means that for each surface interaction we want to determine the radiance $L_{\mathrm{r}}\left(\boldsymbol{x}, \boldsymbol{\omega}_{\mathrm{r}}\right)$, that is, the radiant flux per unit solid angle emitted or reflected, or transmitted at point $\boldsymbol{x}$ of the surface in direction $\omega_{\mathrm{r}}$.

The radiance consists of the light that is emitted in that direction at the surface point, $L_{e}\left(\boldsymbol{x}, \omega_{\mathrm{r}}\right)$, and the light that is irradiated and reflected at that point. The latter is described by an integral over the positive hemisphere $\Omega^{+}$: for each in- 


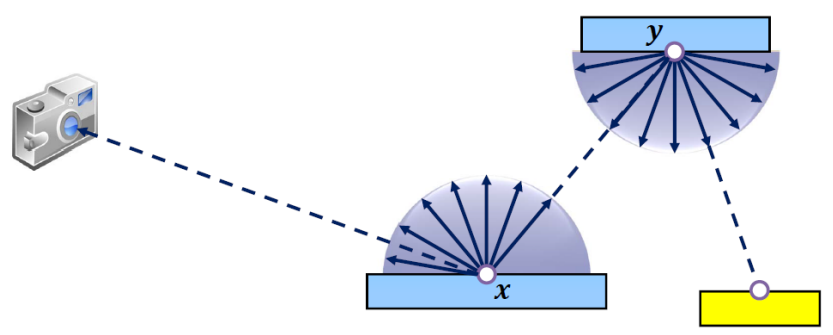

Figure 3. Illustration of distributed ray tracing.

coming direction $\omega_{i}$ we project the solid angle to the surface and multiply it by the irradiance $L_{i}\left(\boldsymbol{x}, \omega_{i}\right)$ reaching the point $\boldsymbol{x}$ from direction $\boldsymbol{\omega}_{i}$ and by a function that describes the reflectance, that is, the ratio between irradiance and radiance; cf. Venable and Hsia (1974). Typically, this is a bidirectional reflectance distribution function (BRDF) $f_{\mathrm{r}}\left(\boldsymbol{\omega}_{\boldsymbol{i}}, \boldsymbol{x}, \boldsymbol{\omega}_{\mathbf{r}}\right)$, introduced by Nicodemus et al. (1977).

All terms combined lead to Eq. (1), also called the rendering equation, which can be evaluated recursively in order to compute the illumination of a scene (Kajiya, 1986).

$L_{\mathrm{r}}\left(\boldsymbol{x}, \boldsymbol{\omega}_{\mathrm{r}}\right)=\int_{\Omega^{+}} f_{\mathrm{r}}\left(\boldsymbol{\omega}_{i}, \boldsymbol{x}, \boldsymbol{\omega}_{\mathrm{r}}\right) L_{i}\left(\boldsymbol{x}, \boldsymbol{\omega}_{\boldsymbol{i}}\right) \cos \theta_{i} \mathrm{~d} \boldsymbol{\omega}_{\boldsymbol{i}}$

Monte Carlo (MC) methods are a broad class of algorithms that numerically evaluate an integral by repeated random sampling. They are suitable for high-dimensional problems, and as such a good choice to compute the value of the surface integral, as ray tracing is a high-dimensional problem because of multiple reflections and the recursive nature of the rendering equation. The Monte Carlo evaluation of the rendering equation leads to approximation Eq. (2).

$L_{\mathrm{r}}\left(\boldsymbol{x}, \omega_{\mathrm{r}}\right) \approx \frac{4 \pi}{N} \sum_{i=0}^{N-1} f_{\mathrm{r}}\left(\omega_{i}, \boldsymbol{x}, \omega_{\mathrm{r}}\right) L_{i}\left(\boldsymbol{x}, \omega_{i}\right) \cos \theta_{i}$

Markov chain Monte Carlo (MCMC) methods sample from a probability distribution based on a Markov chain. This allows the construction of a Markov chain with a desired distribution. Also, in the context of rendering, Markov chains make it possible to construct new paths by mutation of existing paths, a fact that is exploited by the Metropolis light transport (MLT) that is described in Sect. 2.3.7.

\subsection{Comparison of the suitability of (Markov chain) Monte Carlo rendering techniques for $\mathrm{AOI}$}

(MC)MC rendering methods are the predominant way to compute light transport simulations nowadays. In all their diversity, they all share the concept of stochastically creating paths, connecting the sensor to the light sources. In this section, we discuss different (MC)MC rendering algorithms and sampling strategies suitable for the simulation of measuring situations. As mentioned in introductory Sect. 1 the



Figure 4. Illustration of path tracing.

main differences of these approaches lay in the path sampling strategies.

\subsubsection{Distributed ray tracing}

Ray tracing is the basic approach of casting rays starting from the camera and recursively casting successive rays on reflections. Recursive evaluation of the rendering equation, described in Sect. 2.2, by stochastically sampling the integral leads to distributed ray tracing, introduced by Cook et al. (1984). On each surface interaction $N$ rays are followed, as illustrated in Fig. 3. Sadly, this leads to exponential growth.

Summary. Distributed ray tracing is the straightforward implementation of recursive Monte Carlo evaluation of the rendering equation and as such easy to implement, but more recent methods do not suffer from exponential growth.

\subsubsection{Path tracing and next event estimation}

Path tracing, introduced by Kajiya (1986), improves upon distributed ray tracing by tracing whole paths from camera to light sources. That is, at every surface interaction only a single new direction is sampled so that each time a single, unambiguous path is generated, and of those paths we create $N$ for each pixel of the resulting image, as illustrated in Fig. 4.

One problem of ray tracing and path tracing is that a ray or path that does not hit a light source transports no energy and thus has no contribution to the image pixel. At the same time, it is unlikely to hit a light source, in general. To alleviate this problem, optionally, next event estimation (NEE, Kajiya, 1986) can be used to directly sample light sources.

While path tracing in most cases also converges without NEE, with NEE paths that reach light sources, and thus transport much energy, are generated explicitly and therefore in general earlier. This means that images of early stages of the rendering give a better impression of the illumination of the scene. Thus, NEE is also a good method in case rough preview images are useful. 




Figure 5. Illustration of light tracing.

Path tracing is able to render caustics ${ }^{1}$ as long as the light source has finite area (i.e., not a point light source). However, the corresponding transport paths are typically sampled with low probability. Thus, path tracing is not well suited for handling such situations.

Summary. Path tracing is easy to implement and suitable if there are no caustics from small light sources and if direct connections to the light sources are possible. Therefore, path tracing is a good candidate for measurement setups where objects are recorded in transmitted light, as here the light source is usually big and always directly visible.

\subsubsection{Light tracing}

Light tracing, sometimes also referred to as backward ray tracing (Arvo, 1986), is the complementary method of path tracing: as in reality, paths start from the light sources and are followed until they hit the image plane, i.e., the simulated image sensor of the virtual camera. Directions are sampled as is done for path tracing. See Fig. 5.

Next event estimation (NEE) is also optionally possible; in this case, connections to the camera are sampled. However, for light tracing NEE is more problematic in the context of specular surfaces and lens models: specular surfaces limit the reflection path to just one direction. Therefore, it is not possible to sample a different direction that would hit the sensor. Obviously, this is the case when the virtual camera contains a lens system in front of the sensor.

Summary. Like path tracing, light tracing is easy to implement, but it only works well if the light sources directly emit to the surfaces visible to the camera. Otherwise, path tracing (or forward ray tracing) is still employed to determine visual surfaces, as in the next method.

\subsubsection{Bi-directional path tracing (BDPT) and multiple importance sampling (MIS)}

Bi-directional path tracing, introduced by Lafortune and Willems (1993), is a combination of path and light tracing: the subpaths are constructed starting from both ends, cam-

\footnotetext{
${ }^{1} \mathrm{~A}$ caustic, in optics, is a light bundling pattern created by objects or materials focusing or diverting light by refraction or reflection.
}

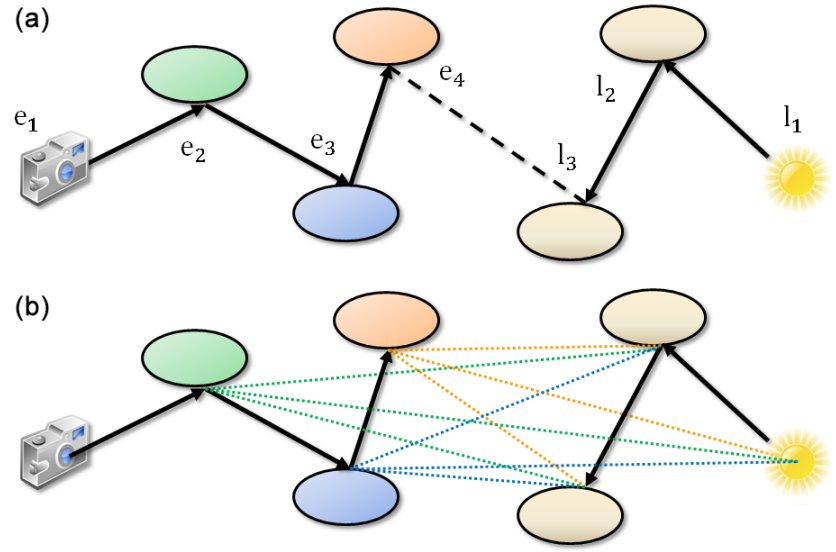

Figure 6. Illustration of bi-directional path tracing (BDPT). (a) "Eye" subpaths $e_{i}$ starting at the camera and "light" subpaths $l_{i}$ (b) deterministically connect all intermediate paths (nodes).

era and light sources; the intersections of paths with objects of the scene are called nodes. In addition to direct sampling of light sources and the camera, respectively, the intermediate nodes of both path and light tracing subpaths can be deterministically connected, greatly increasing the number of resulting paths that are likely to transport much energy, and thus have a lot of influence on the illumination of the simulated scene. See Fig. 6.

However, increasing the number of possible path combinations also means that a technique is necessary to keep the variance of the Monte Carlo estimation low, as the reuse of "light" and "eye" subpaths in the path combinations results in high variance (apparent as high-frequency noise in the resulting image) due to correlation between the paths (Popov et al., 2015). Therefore, BDPT usually requires variance reduction via multiple importance sampling (MIS) to be practical, which unfortunately makes this method non-trivial to implement. MIS, introduced by Veach (1998), is a technique to use multiple sampling methods to evaluate an integral and combine the sample values in a provably good way.

Summary. As mentioned, BDPT is non-trivial to implement because it needs MIS to be practical. BDPT works well in scenes where the deterministic connections are not blocked. This should usually be the case for AOI settings as the measurement setup is usually designed in a way that the objects are well lit and visible to the camera, that is, the objects are visible to both camera and light source.

\subsubsection{Many-light methods (variants of BDPT)}

The many-light methods originate from the instant radiosity algorithm proposed by Keller (1997). They are a variant of BDPT where light subpaths are created as before, but now the nodes are treated as so-called virtual point light (VPL) sources. This means that at the location of the nodes from the light subpath new light sources are inserted into the scene. 
Summary. The many-light approach works very well for mostly diffuse scenes and is easy to implement. On the downside, it has problems with glossy surfaces and is even impossible to use with specular surfaces; cf. Dachsbacher et al. (2014).

\subsubsection{Photon mapping}

Photon mapping is the somewhat confusing yet widely used short name for Global Illumination using Photon Maps introduced by Jensen (1996). In photon mapping light subpaths are also created, but in this model, the light sources send out photons, i.e., energy units. The node locations, that is, locations where energy impinges on the surface, are recorded in a data structure called a photon map; cf. Fig. 7a.

Afterwards, the scene is rendered with path tracing, but instead of treating the nodes of the light paths as VPLs as in the many-light methods and directly connecting to them with NEE, the photon map is used to compute the illumination. That is, the energy at a surface point is estimated by counting the photons in the local environment of the intersection point (density estimation); cf. Fig. 7b.

Summary. Photon mapping works well with diffuse surfaces and can render caustics efficiently. For glossy surfaces, it (gracefully) degrades to path tracing. Photon mapping can be made robust and has the advantage of producing images with low noise levels, but the density estimation also causes a bias (systematic error); cf. Hachisuka and Jensen (2009).

More extensions and variants to bi-directional methods exist, such as, e.g., Georgiev et al. (2012).

\subsubsection{Metropolis light transport (MLT)}

The family of the Metropolis light transport methods use the Metropolis-Hastings (MH) algorithm, introduced by Hastings (1970), to sample the space of all possible light transport paths, and as such they all are Markov chain Monte Carlo (MCMC) methods.

The basic idea is to first find an important path, that is, a path that transports much energy and thus has high influence on the resulting image, and then to generate similar paths by mutation of the existing important path. This is one of the best strategies for difficult situations, such as, for example, the scene illustrated in Fig. 8, in which the only light source is concealed without any direct connections to the camera.

Numerous variants exist, e.g., sampling in primary space (Kelemen et al., 2002) or in path space (Veach, 1998), as well as modern extensions such as energy redistribution path tracing (ERPT, Cline et al., 2005), manifold exploration (Jakob and Marschner, 2012), half-vector space transport (Kaplanyan et al., 2014), and gradient domain methods (Kettunen et al., 2015).

Summary. MLT methods have in common that they are very powerful in exploring difficult light transport phenomena (e.g., caustics). However, they have to be initialized re-
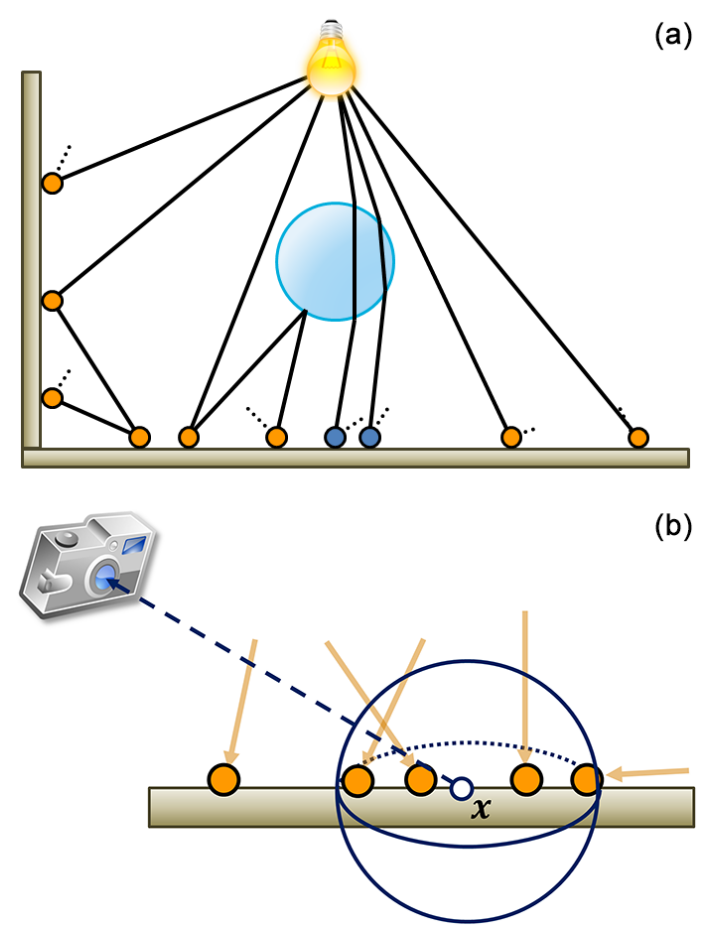

Figure 7. Illustration of photon mapping. (a) Light sources send out energy as photons; (b) a density estimation is used to estimate the energy per surface area.

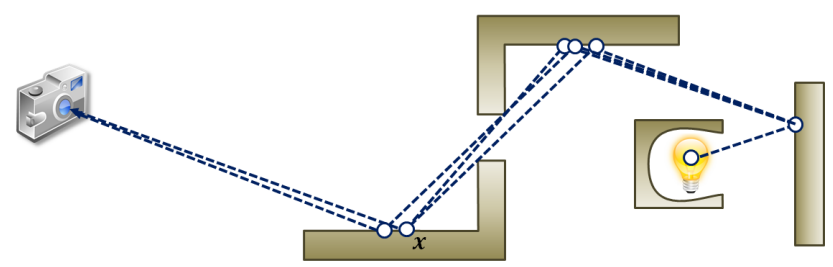

Figure 8. Illustration of Metropolis light transport (MLT) with one important path that reaches the single, concealed light source of this difficult scene, and two mutations of this path.

peatedly with independent MC samplers (e.g., BDPT) and thus rely on those to detect actual occurrences of said light phenomena. In image rendering, this results in images where individual components are rendered with little noise but all occurrences of the light phenomena are only found over time. Also, MLT methods share the property that they are difficult to implement (an exception is Kelemen et al., 2002) and difficult to control.

\subsubsection{Realistic lenses}

In interactive image synthesis, simple perspective projection is often used that corresponds to the projection of a pinhole camera. But rendering systems also often use simplified approaches such as the pinhole camera or the thin lens model. While it is possible to just include even a complex lens in the 


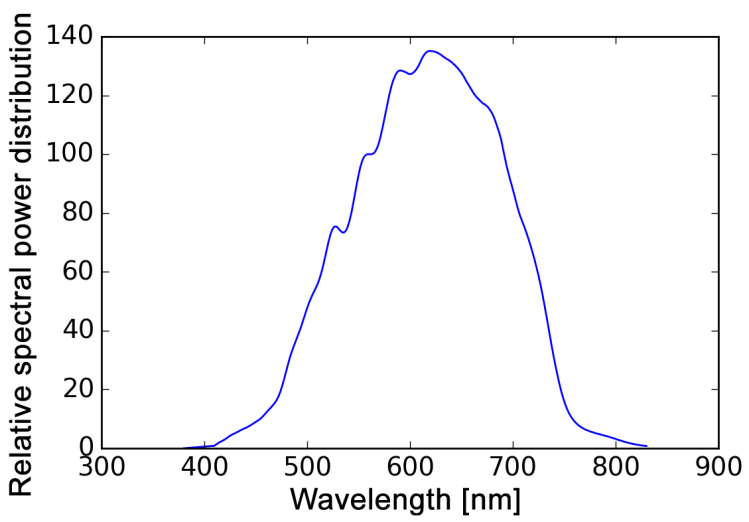

Figure 9. Emission spectrum of a tungsten halogen lamp (Model 3900 by Illumination Technologies, Inc.).

virtual scene and trace rays through it, this leads to very inefficient rendering, as, for straightforward ray tracing, $95 \%$ of all ray samples, or more, might not leave a real-world lens system and enter the actual scene (Hanika and Dachsbacher, 2014).

The reason for this lies in the fact that straightforward ray or path tracing starts at the sensor and will simply sample locations on the image sensor and directions off the sensor to generate rays, and, thus, many of the rays will hit the housing of the camera or the aperture. This can be avoided by implementing importance sampling (cf. Sect. 2.3.4) of the aperture, that is, by not only sampling the sensor but also points on the aperture. It has to be noted that sampling the aperture is usually not easily possible, as the aperture stop is typically located within the lens system, i.e., between optical elements; refer to Hanika and Dachsbacher (2014) and Schrade et al. (2016) for a detailed analysis of this problem.

\section{Realistic image formation to simulate AOI systems}

\subsection{Synthetic images in the context of optical inspection}

In Retzlaff et al. (2015) we describe the idea of using computer graphics methods to allow systematic and thorough evaluation of automated optical inspection (AOI) systems. Instead of using real objects and acquisition systems, computer graphics methods are used to create large virtual sets of samples of test objects and to simulate image acquisition setups. We use procedural modeling techniques to generate virtual objects with varying appearance and properties, mimicking real objects and sample sets. Physical simulation of rigid bodies is deployed to simulate the placement of virtual objects, and using physically based rendering techniques we create synthetic images. These are used as input to an AOI system instead of physically acquired images. This enables the development, optimization, and evaluation of the image processing and classification steps of an AOI system independently of a physical realization.

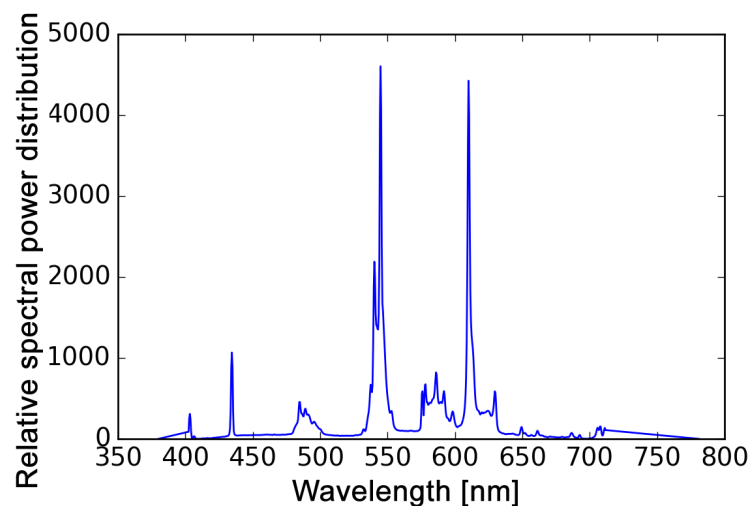

Figure 10. Emission spectrum of a fluorescent ceiling light.

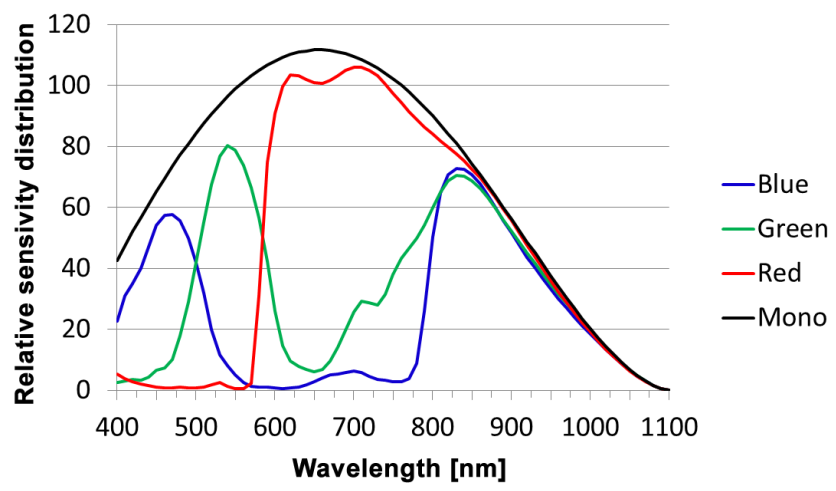

Figure 11. Raw sensor response of the ELiiXA UC4/UC8 line scan camera by e2v.

We demonstrated this approach for shards of glass, as sorting glass is one challenging practical application for AOI. In this paper, we focus on the aspects of image synthesis.

\subsection{Synthetic images of glass shards using rasterization}

\subsubsection{Efficient generation of synthetic glass shards}

Retzlaff et al. (2015) describe a procedural model for the generation of virtual 3-D models of glass shards based on an algorithm described by Martinet et al. (2004). We modified the algorithm to generate plausible shards with a certain smoothing, as waste glass is repeatedly relocated and this leads to rounded edges of the glass shards. In addition to this, our carving volume is smooth to allow fast intersection, while we achieve plausible breaking edges by displacement mapping of the intersection surfaces using hardware-accelerated tessellation shaders that were introduced in OpenGL 4.0.

These modifications make a shard generation in negligible computation time possible by adding surface detail in real time. 


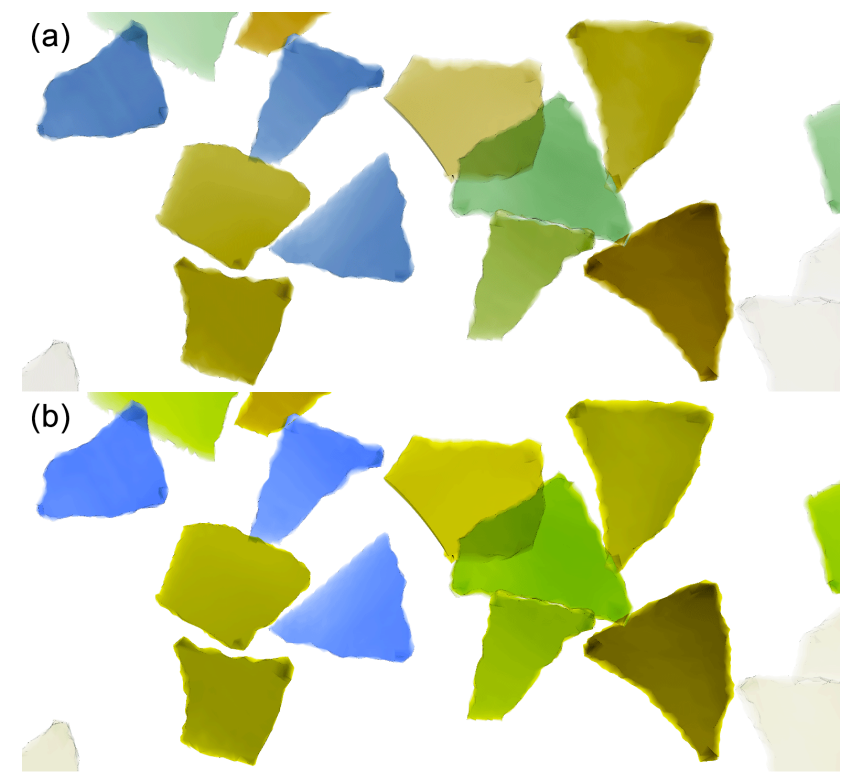

Figure 12. Images rendered in real time using the color matching functions of the CIE 1931 standard colorimetric observer. (a) CIE Illuminant D65; (b) fluorescent illumination of Fig. 10.

\subsubsection{Real-time rendering of shard distributions}

Our implementation includes real-time rendering of shard distributions by hardware rasterization on the GPU using OpenGL 4.2 (Segal and Akeley, 2012).

As glass is an optically semi-transparent material, a method of transparency rendering is necessary. OpenGL itself only provides alpha blending that can be used to render transparent materials. But rendering transparency using alpha blending implies rendering the objects in a sorted order. As sorting for every rendered frame is impractical and even not always possible (e.g., for mutually overlapping objects), order-independent transparency rendering (OIT) techniques have been invented.

Our previous implementation as presented in Retzlaff et al. (2015) used depth peeling (introduced by Cass, 2001), a robust hardware-accelerated OIT method, but meanwhile we replaced this with an implementation of a more powerful OIT technique making use of per-pixel concurrent linked lists (introduced by Yang et al., 2010). OIT rendering using depth peeling can store only a quite limited number of material interactions per image pixel requiring multiple rendering passes in case of many interactions. Per-pixel linked list OIT, on the other hand, allows one to store a high number of interactions in a single rendering pass, and the realistic rendering of the breaking edges of glass shards may require a high number of interactions as the edges can be quite rough.

As our previous publications Retzlaff et al. (2015) and Retzlaff et al. (2014) focus on the generation of realistic virtual objects and scenes based on measured data as well as the methodical procedure of using synthetic images for the eval-

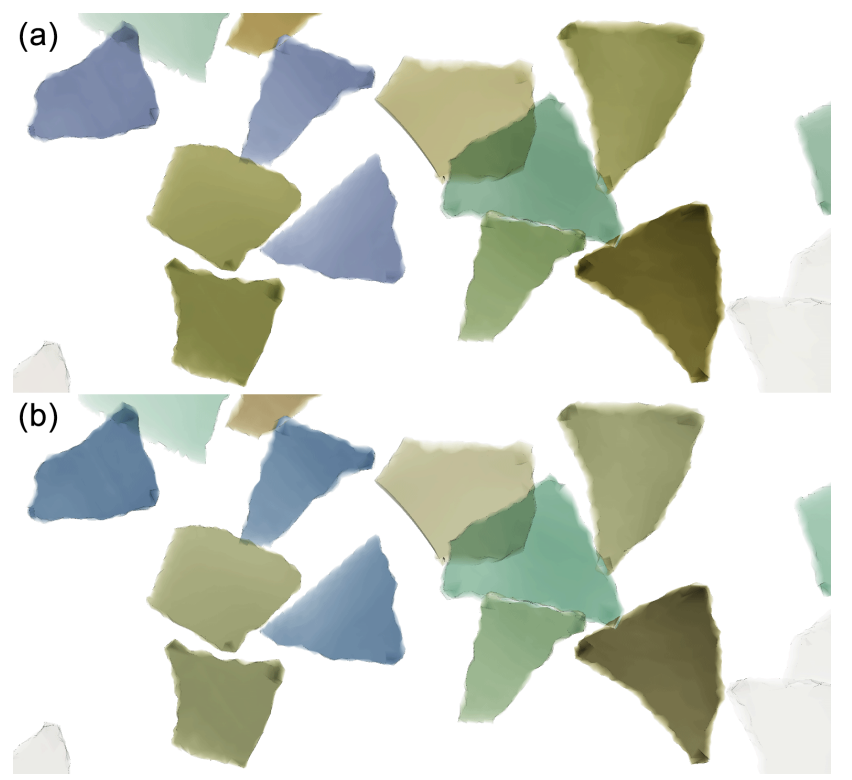

Figure 13. Images rendered in real time using the ELiiXA sensor sensitivity function depicted in Fig. 11. (a) and (b): images illuminated as in Fig. 12.

uation of real AOI systems, we had used simplified models of the illumination and image acquisition for the image synthesis, e.g., ideal sensors and simplified optical image formation.

Most importantly, we have used RGB rendering. The synthesis was therefore reduced to only three values describing red, green, and blue norm stimuli, and could not reproduce spectral effects such as dispersion.

\subsubsection{Replicating real image acquisition systems and spectral rendering}

Recently, we have enhanced our real-time method to support spectral rendering and to replicate more physical aspects of real image acquisition systems; that is, we now support the simulation of real light sources and sensor responses of real image sensors.

Of course, spectral rendering is especially important in the context of color filters that limit the light spectrum to a narrow spectral range combined with lighting by a non-uniform illumination.

Light emission and transport we now describe as full spectra. That is, we use the spectral data of real, measured light sources. The light interaction is computed using measured absorption spectra (of course, for other use cases reflection spectra are equally possible). The resulting spectral power distribution is multiplied by a color matching or sensor sensitivity function, as depicted in Fig. 11. Integration across the spectrum leads to the intensity values of each sensor sensitivity function, usually an RGB triplet in the RGB space of the simulated sensor that can be displayed. 




Figure 14. A synthetic image of procedurally generated virtual glass shards on a diffuse reflecting surface generated by our Monte Carlo rendering framework.

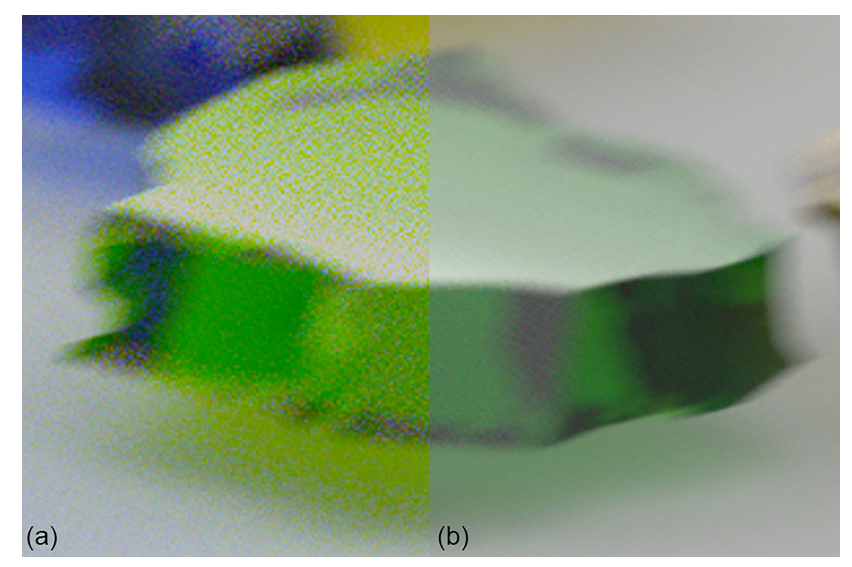

Figure 15. Close-up view of a synthetic shard. (a) Fluorescent illumination with bright spectral lines, as depicted in Fig. 10; (b) CIE Standard Illuminant D65. Both images are rendered with 2048 samples per pixel.

In the case of the CIE color matching functions (Smith and Guild, 1931-1932) $X Y Z$ coordinates are computed that describe the color perception of a human being as standardized by the CIE 1931 standard colorimetric observer. The $X Y Z$ images can be converted to an RGB working space, such as, for example, the sRGB color space (IEC, 1999), and displayed on a calibrated screen. It thus can serve as a reference image for an expert user designing an AOI setup, as it corresponds to the color impression one would perceive in the simulated viewing conditions; cf. Fig. 12.

Simulation of chromatic adaptation can account for the difference in white reference of the simulated light emission and of the computer screen that is used for viewing.

For our real-time implementation we use a binning approach to spectral rendering. That is, the full spectrum of the visible light is quantized into bins of a certain width; thus, the spectra are approximated by a step function. The spectra can either be approximated by equal width bins, which is a sufficient approximation for smooth spectra, or it can be

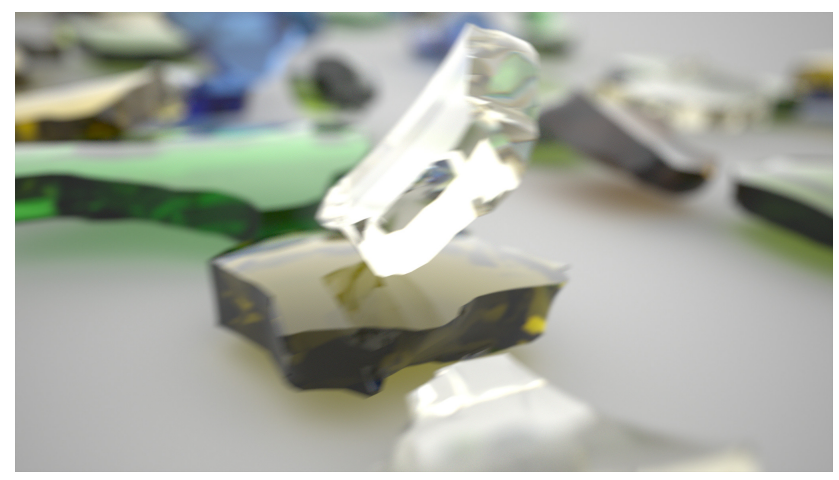

Figure 16. A close-up view of the scene also displayed in Fig. 14 from a different angle.

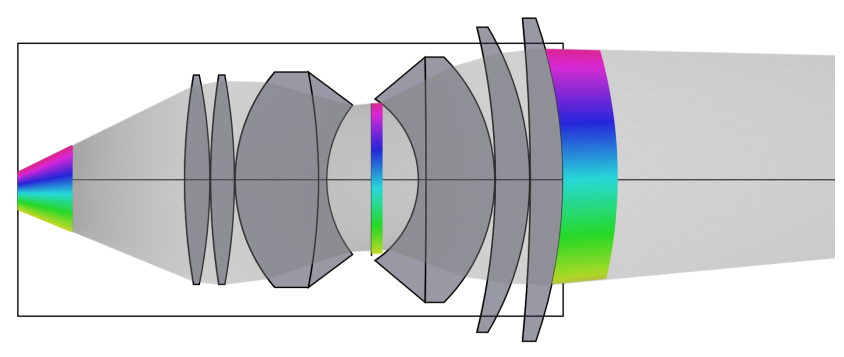

Figure 17. Double-Gauss lens by Angénieux (1955).

adaptive, as is necessary in the case of illumination with light depicting narrow bright peaks.

\subsubsection{Light sources with bright spectral lines and results}

While light sources such as incandescent light bulbs or halogen lamps have quite smooth spectra, see Fig. 9, the spectra of other light sources such as, for example, fluorescent lamps exhibit a number of narrow bright peaks, as depicted in Fig. 10. Such a lighting condition in combination with certain sensors can result in images in which actual spectral differences cannot be perceived or easily detected anymore.

This can be simulated with spectral rendering, and helps to design an acquisition setup that does not exhibit such problems by choosing a suitable combination of sensor and illumination; see Fig. 13 and compare to Fig. 12.

Both image sets have been generated with our real-time shard generation and rendering implementation, and demonstrate the capabilities of this approach. While Fig. 13 has been rendered using the sensor sensitivity function of a physical camera, as depicted in Fig. 11, Fig. 12 shows the same virtual glass shard scene with a color impression as a human according to CIE 1931 would perceive it in the same simulated viewing conditions. 


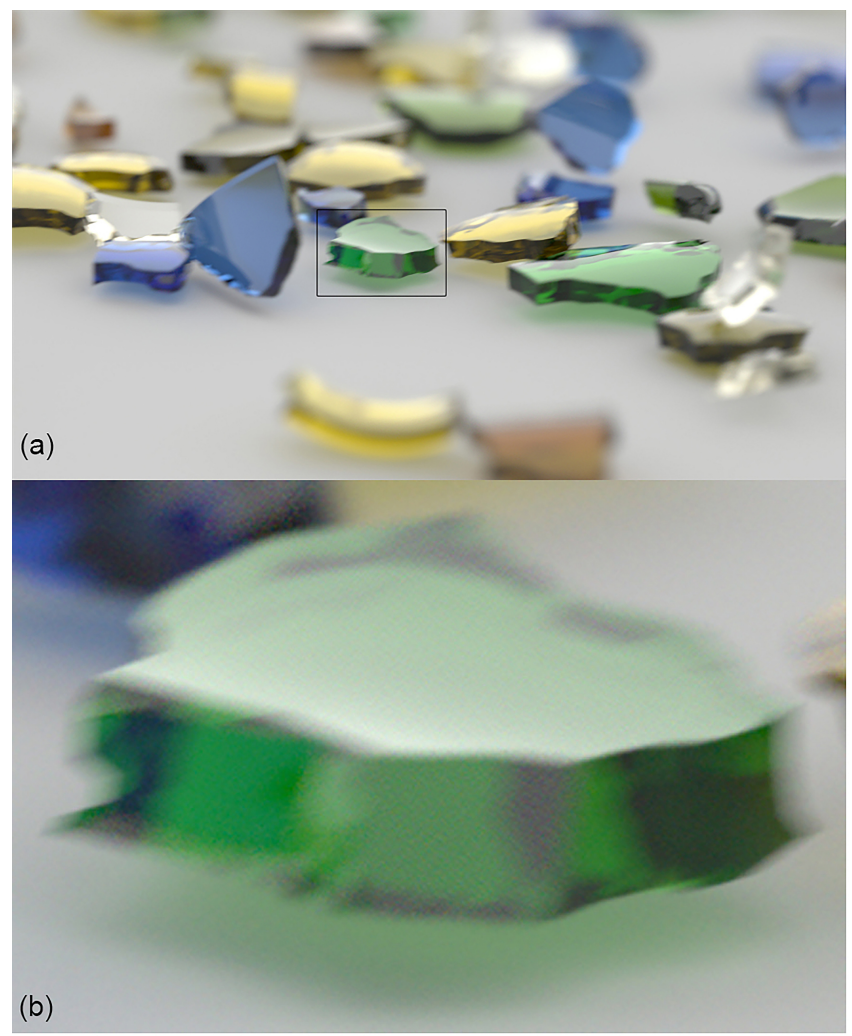

Figure 18. (a) Synthetic image and enlarged section (b) rendered using a thin lens model exhibiting a narrow depth of field (DOF).

\subsubsection{Limits of the rasterization approach}

While it is possible to add support to rasterization for handling more complex light interaction phenomena such as, for example, refraction and dispersion - and has in fact been realized in our simulation in the form of a proof of concept implementation for light refraction - one has to accept that rasterization has its limits and at a certain point a ray tracing approach begins to be more feasible, and even more efficient.

Support for refraction can be added to a rasterization approach by ray marching, that is, by determining the intersection point of a ray with a surface not analytically, but by iteratively taking steps along the ray and checking each time whether an intersection has taken place. Obviously, it is much more straightforward to add light refraction to a ray tracer. Dispersion constitutes a similar case. But the aforementioned point is surely exceeded when global illumination or simulation of real lens systems including monochromatic and chromatic aberration is asked for. In these cases, a more efficient approach for ray casting than ray marching is called for, as demonstrated in the next part of this section.

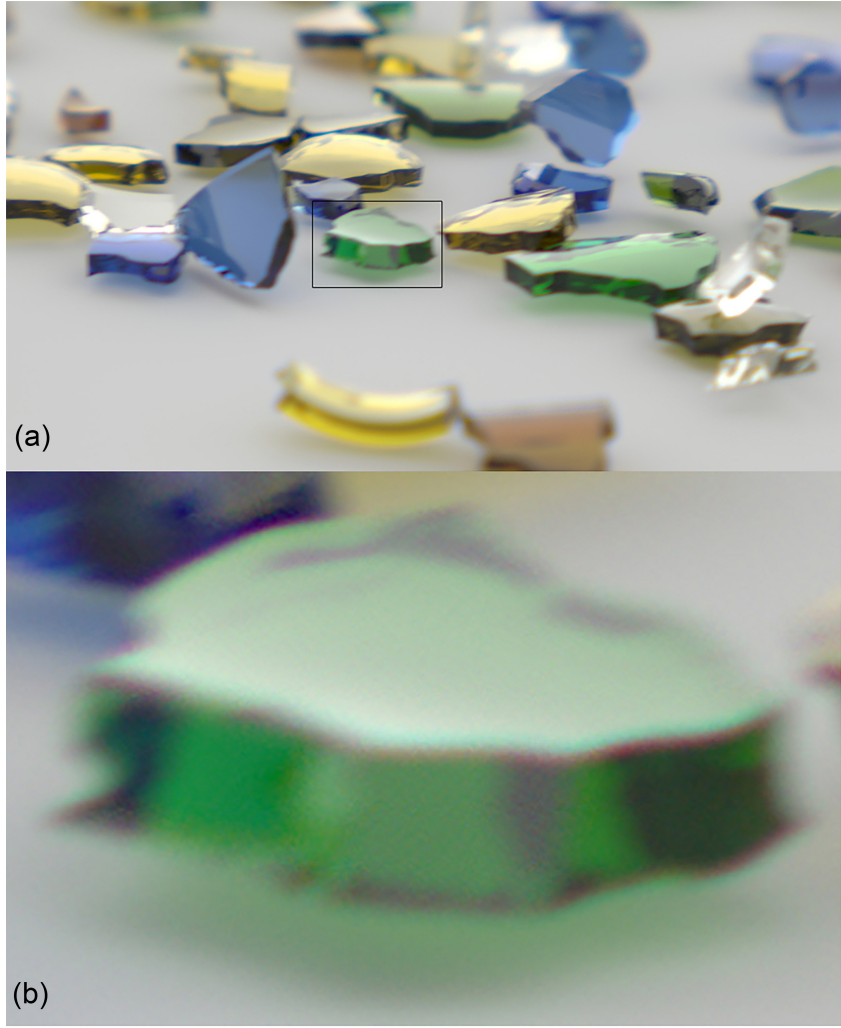

Figure 19. Same scene as in Fig. 18 but using a simple achromatic doublet lens (Edmund Optics \#NT32-921), exhibiting a less narrow DOF but strong chromatic aberration (color fringing).

\subsection{Synthetic images of glass shards using physically based image synthesis}

\subsubsection{Revised generation of synthetic glass shards}

There is a downside to the efficiently generated virtual glass shards that we described in Sect. 3.2.1. In the case of considerable displacement, the polygonal mesh might get selfintersections or holes at the transition between intersection surface and original shard surface. Small irregularities can be fixed easily, but bigger defects would require a remeshing of the polygon mesh, slowing down the generation considerably. Such erroneous meshes describe surfaces that are not physically possible, and thus present problems for physically based rendering methods. As a result, the law of conservation of energy might get violated at such irregularities.

Therefore, we vouched for a new method of generating shards of broken glass, dropping the requirement of real-time generation and instead introducing a pool of precomputed shard meshes.

Most existing fracturing methods in the field of computer graphics are based on Voronoi partitions or tessellations, but many implementations generate only undetailed, flat intersection surfaces that are also not well tessellated and thus not suitable for surface perturbation without remeshing. An 


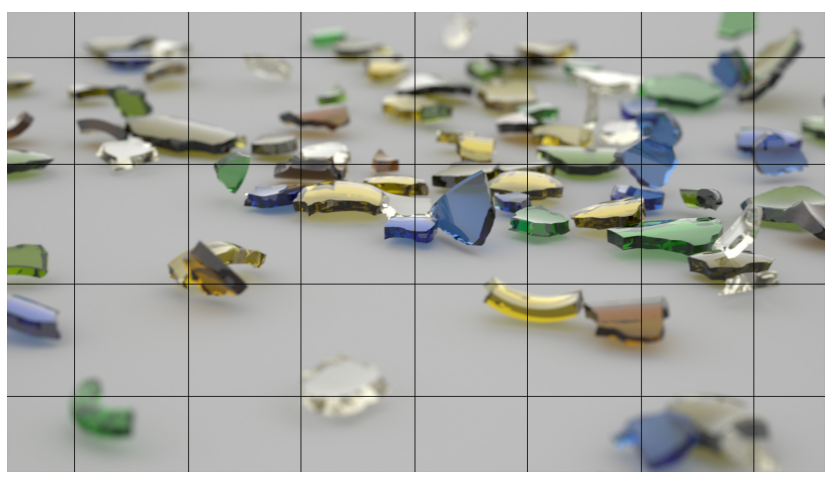

Figure 20. Synthetic image using the simple thin lens model as perceived by the CIE 1931 standard colorimetric observer, CIE D65 hemispherical illumination.

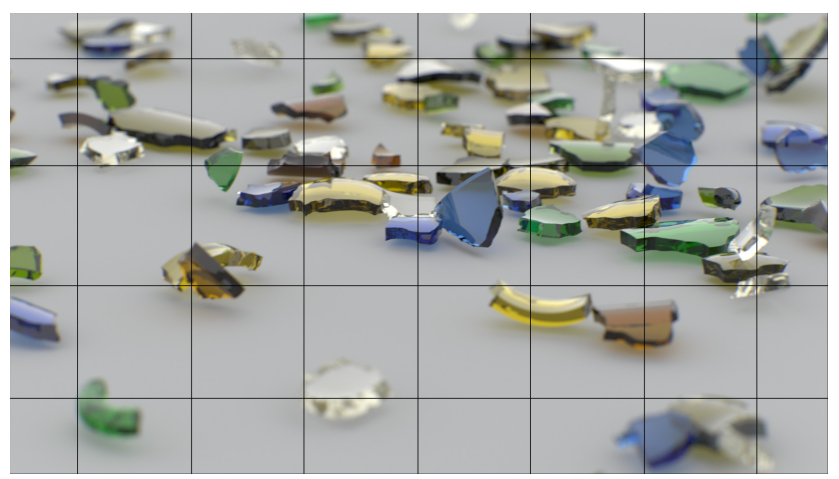

Figure 21. Same as in Fig. 20 but using the Double-Gauss lens by Angénieux (1955), also depicted in Fig. 17. The black grid is overlaid to make the difference in distortion easily recognizable.

example of this is the Cell Fracture implementation of the Blender 3-D modeling software by the Blender Foundation.

One notable exception is the method introduced by Korndörfer (2011). It is also based on Voronoi partitioning, but uses a 3-D voxel grid and puts emphasis on achieving a regular quad meshing of the boundary surface. The Voronoi regions are not based on the Euclidean distance, but instead on the length of paths in a graph defined by the voxels. The shape of the regions can be controlled by weights of the graph edges. The method already supports random noise edge weights in order to generate irregular, i.e., plausible, surface structures, making it a suitable candidate for our task of generating virtual glass shards.

\subsubsection{Physically based rendering and resulting images}

Figure 14 shows a scene of the shards generated with the method of Korndörfer (2011) and rendered using our existing Monte Carlo ray tracing framework supporting global illumination, that is, a physically based simulation of light transport, which inherently accounts for phenomena such as reflections and shadows. We have chosen path tracing even

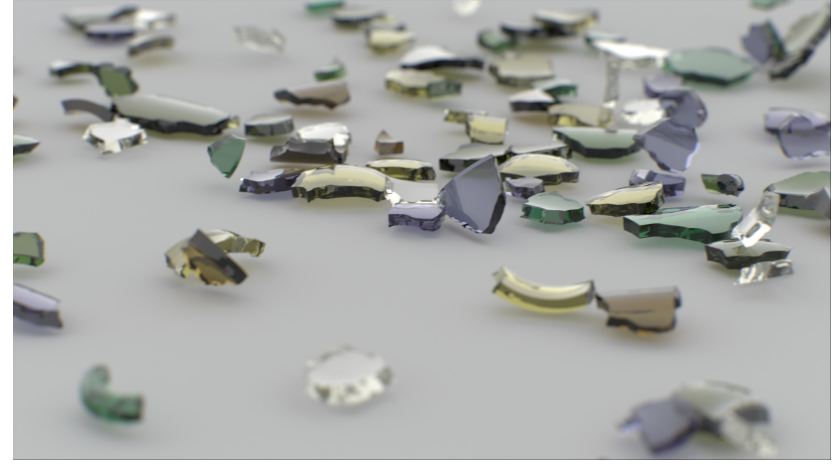

Figure 22. Same as in Fig. 21 but detected by the ELiiXA sensor, depicted in Fig. 11.



Figure 23. Same as Fig. 21 but with motion blur.

without NEE for our task, as the AOI setup exhibits a very large light source.

As the ray tracing framework does not use spectral binning but instead supports full spectral rendering with Monte Carlo spectral sampling, it can simulate dispersion. Figure 16 shows a close-up view of the same scene as in Fig. 14 (from a slightly different angle), exhibiting light refraction and chromatic dispersion.

It is interesting to note that the spectra of the illumination and interacting surfaces might also affect the rendering time or image quality. Uniform sampling of the emission spectrum leads to noisy images in the case of spectra with bright narrow peaks compared to images using a more homogeneous spectral illumination, as samples fall equally on every part of the spectrum. This is illustrated in Fig. 15.

Importance sampling that results in a higher sampling rate in regions with higher radiance while still being an unbiased estimation can leverage this problem without increasing the sampling count (and thereby increasing the rendering time).

\subsubsection{Adding realistic lenses}

We use our method and implementation presented in Hanika and Dachsbacher (2014) to support efficient rendering using 


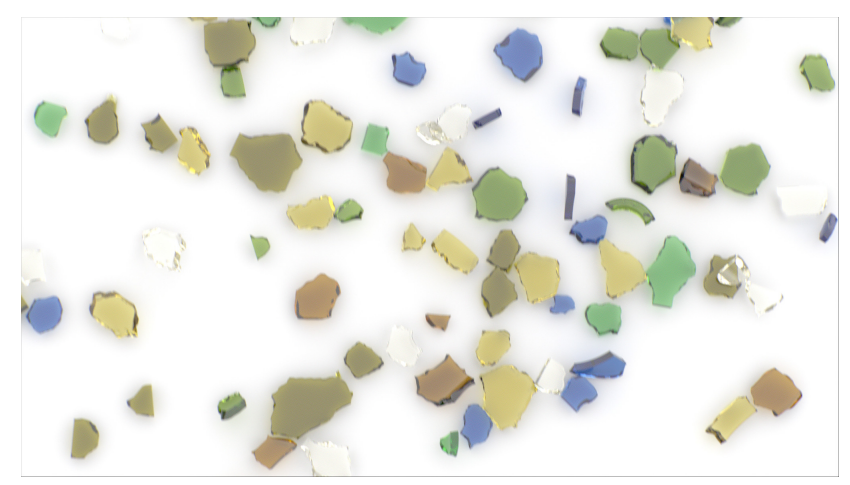

Figure 24. Double-Gauss lens (Angénieux, 1955), CIE D65 hemispherical illumination, CIE standard colorimetric observer.



Figure 25. Same as Fig. 24 but detected by the ELiiXA sensor, depicted in Fig. 11.

a wide range of real-world lenses with only negligible overhead compared to rendering with the thin lens model. Such real-world lenses can consist of dozens of lens elements and an aperture, such as, for example, the Angénieux DoubleGauss lens (cf. Angénieux, 1955, US Patent 2701 982, effective focal length $100 \mathrm{~mm}, \mathrm{f} / 1.1$ ), as depicted in Fig. 17. Still, this approach can accurately compute the lens image and thus be used to simulate flaws and shortcomings of real lens systems.

Figure 18 and Fig. 19 show a group of images of the same scene of glass shards on a diffuse reflecting surface but using different lenses, the thin lens model and a simple achromatic doublet lens (Edmund Optics \#NT32-921, modified, effective focal length $129 \mathrm{~mm}, \mathrm{f} / 3.3$ ), respectively. Both lenses have got different depths of field, and the image by the achromatic doublet lens exhibits strong chromatic aberration, commonly referred to as color fringing.

\subsubsection{Results and rendering computation times}

To conclude this section, we present an overview of renderings with different lenses as well as color matching and sensor sensitivity functions, respectively. Figures 20 to 23 show the scene from a flat angle, while Figs. 24 to 27 show a top

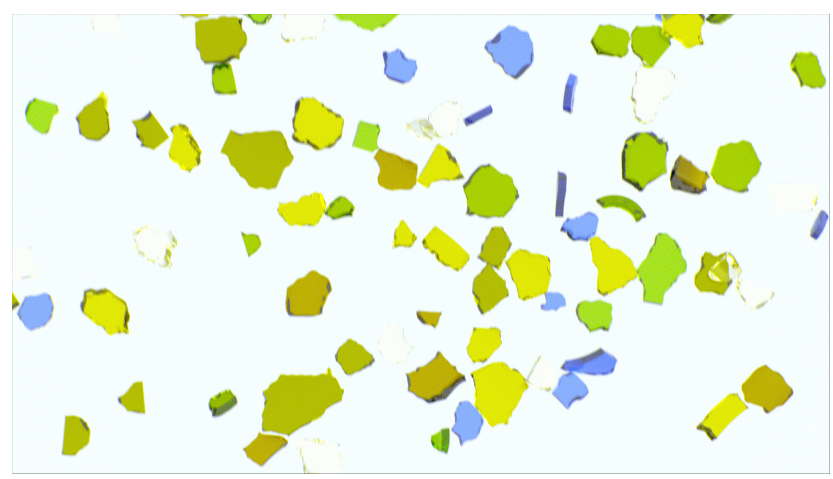

Figure 26. Same as Fig. 24 but with the fluorescent ceiling light of Fig. 10 as strong transmitted light and hemispherical illumination.

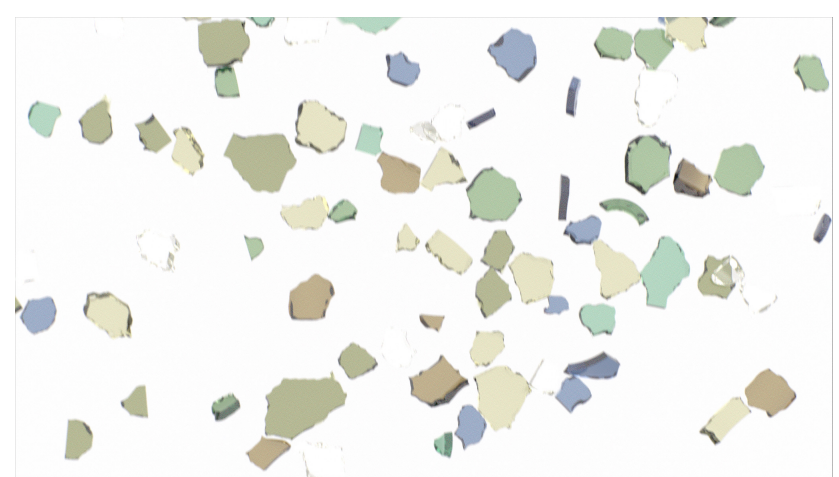

Figure 27. Same as Fig. 26 but with the ELiiXA sensor, depicted in Fig. 11.

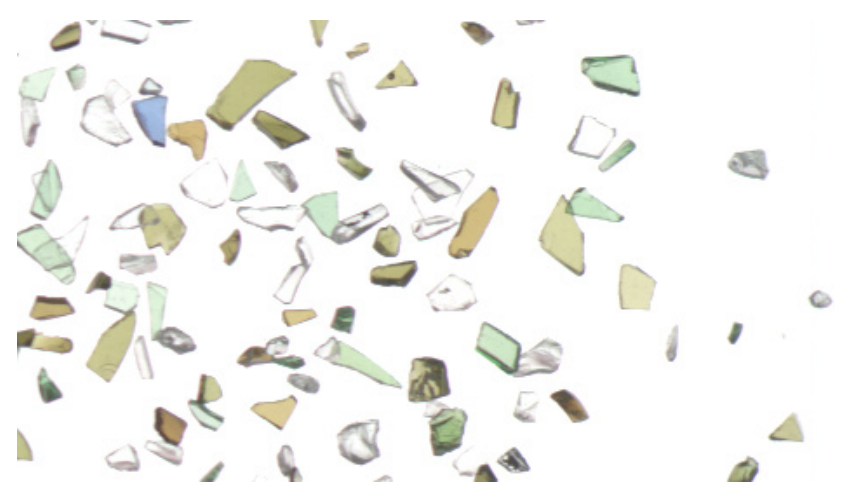

Figure 28. Recorded image of real glass shards obtained by a physical line scan camera (unknown type and sensor) of an AOI image acquisition system (Fraunhofer IOSB, 2012) in transmitted light of a fluorescent illumination, scaled to approximately fit the size of the simulated shards.

view of the same scene resembling the arrangement of the real image acquisition system. Actually, in the real system the shards always appear in a strong transmitted light as in Figs. 26 and 27, and not on a diffuse reflecting surface as in the other images. 
For comparison, Fig. 28 shows a recorded image of real glass shards obtained by a physical line scan camera (unknown type and sensor) of an AOI image acquisition system in transmitted light of a fluorescent illumination. This closely matches the simulated conditions of Fig. 27.

As we generate the virtual scenes using physical simulation of gravity and collisions of rigid bodies, we can easily generate consecutive scenes separated by small time differences, and thus also render images with motion blur of the shards sliding along a sloping surface, as can be seen in Fig. 23.

Tables 1 and 2 contain the computation times for the image series Figs. 20 and 23 and Figs. 24 and 27, respectively. All of these images have been rendered at a resolution of $2048 \times 1152$ pixels using simple path tracing without next event estimation (NEE) as the rendering method and 2048 samples per pixel on an Intel Xeon E7-8870 CPU with 10 cores (plus Hyper-Threading) at $2.4 \mathrm{GHz}$.

Please note that we configured our rendering implementation with fixed settings of 2048 samples per pixel regardless of the actual variance to ensure high quality; an adaptive approach could result in shorter rendering times.

The rendering durations are roughly on the same scale, with the exception of Figs. 26 and 27. The scenes of these two images are illuminated with a fluorescent light not only as hemispherical illumination, but also in strong transmitted light to simulate the image acquisition conditions of the physical setup.

These exceptions amply demonstrate that path tracing is a well-suited rendering method for applications in transmitted light. The background consists of a large light source that is easy to hit; therefore, direct connections to the light source are easily possible, while in the case of a diffuse surface the paths that hit it need at least one more path segment to reach the hemispherical surrounding light.

Thus, recording images of the shards for this measurement problem in transmitted light is not only an advisable choice for the subsequent image processing task as the shards appear more clearly in the resulting images without casting shadows on the background; see Fig. 27 and compare to Fig. 25. Also, synthetic images of scenes in transmitted light are faster to compute.

\section{Conclusion and future work}

In summary, we described an entire image synthesis pipeline including all relevant components as well as their efficient and accurate implementation with regard to the simulation and validation of optical measuring systems.

In addition to a real-time image pipeline based on hardware rasterization on the GPU, including spectral rendering using a binning approach, we implemented a second pipeline using physically based simulation of light transport including efficient simulation of real-world lens systems. Altogether,
Table 1. Computation times for image series 1: variation in lens model, Figs. 20 to 23.

Note: the rendering implementation has been configured with fixed settings to ensure high quality, and not to achieve fast computations.

\begin{tabular}{ll}
\hline image & $\begin{array}{l}\text { duration } \\
\text { (hh:mm:ss) }\end{array}$ \\
\hline Fig. 20, thin lens model & $01: 18: 16$ \\
Fig. 21, Angénieux Double-Gauss (DG) & $01: 27: 54$ \\
Fig. 22, Angénieux DG + motion blur & $01: 28: 11$ \\
Fig. 23, Angénieux DG + ELiiXA sensor & $01: 27: 51$ \\
\hline
\end{tabular}

Table 2. Computation times for image series 2: variation in illumination and sensor, Figs. 24 to 27.

Again not optimized for fast computations.

\begin{tabular}{ll}
\hline image & $\begin{array}{l}\text { duration } \\
\text { (hh:mm:ss) }\end{array}$ \\
\hline Fig. 24, CIE D65 + CIE observer & $01: 16: 31$ \\
Fig. 25, CIE D65 + ELiiXA sensor & $01: 16: 13$ \\
Fig. 26, strong Fluorescent + CIE observer & $00: 41: 10$ \\
Fig. 27, strong Fluorescent + ELiiXA sensor & $00: 41: 23$ \\
\hline
\end{tabular}

this resulted in a realistic simulation of the image formation of an automated optical inspection (AOI) image acquisition system.

Meanwhile, in Retzlaff et al. (2016), we investigated the combination of our synthetic image acquisition (SIA) and machine learning to accelerate design and deployment of AOI systems. We demonstrated that a classifier can be trained using synthetic images, and on physically acquired images this classifier performed on par with a classifier that was trained using physically acquired images.

\subsection{Outlook}

The simulated image formation now includes measured spectral light sources, complex light-matter interaction using measured absorption spectra, and realistic lens systems, and reproduces the sensor response of real sensors. One last thing that is left, is the simulation of sensor noise.

The EMVA 1288 Standard for Machine Vision (Jähne, 2010) describes a model for sensor noise. The noise is dissected as a combination of photon noise or, more generally, shot noise that can be described as the square root of the signal, of dark noise governed by the dark current of thermally generated electrons, and of sensor readout noise. This model can be used to simulate the sensor noise of real sensors, so that the resulting synthetic images could finally be deemed "sensor realistic", a term that we use to describe images that comprise the whole image acquisition situation of a physical system so that they can just replace images coming from a real camera. 
Data availability. No data sets were used in this article.

Competing interests. The authors declare that they have no conflict of interest.

Edited by: M. Fischer

Reviewed by: two anonymous referees

\section{References}

Angénieux, P.: Large Aperture Six Component Optical Objective, US Patent 2701 982, issued 15 February, 1955.

Arvo, J.: Backward Ray Tracing, ACM SIGGRAPH '86 Course Notes - Developments in Ray Tracing, 259-263, 1986.

Cass, E.: Interactive order-independent transparency, NVIDIA Corporation, technical report, 2001.

Cline, D., Talbot, J., and Egbert, P.: Energy Redistribution Path Tracing, ACM Trans. Graph. (TOG) - Proc. of SIGGRAPH 2015, 24, 1186-1195, doi:10.1145/1073204.1073330, 2005.

Cook, R. L., Porter, T., and Carpenter, L.: Distributed Ray Tracing, ACM Proc. of SIGGRAPH '84 Computer Graphics, 18, 137145, doi:10.1145/964965.808590, 1984.

Dachsbacher, C., Křivánek, J., Hašan, M., Arbree, A., Walter, B., and Novák, J.: Scalable Realistic Rendering with Many-Light Methods, Computer Graphics Forum, 33, 88-104, doi:10.1111/cgf.12256, 2014.

Georgiev, I., Křivánek, J., Davidovič, T., and Slusallek, P.: Light transport simulation with vertex connection and merging, ACM Trans. Graph. (TOG) - Proc. of SIGGRAPH Asia 2012, 31, 110, doi:10.1145/2366145.2366211, 2012.

Hachisuka, T. and Jensen, H. W.: Stochastic progressive photon mapping, ACM Trans. Graph. (TOG) - Proc. of SIGGRAPH Asia 2009, 28, 1-8, doi:10.1145/1618452.1618487, 2009.

Hanika, J. and Dachsbacher, C.: Efficient Monte Carlo rendering with realistic lenses, Computer Graphics Forum (Proc. of Eurographics), 33, 323-332, doi:10.1111/cgf.12301, 2014.

Hastings, W. K.: Monte Carlo sampling methods using Markov chains and their applications, Biometrika, 57, 97-109, doi:10.1093/biomet/57.1.97, 1970.

Hughes, J. F., van Dam, A., Foley, J. D., and Feiner, S. K.: Computer Graphics: Principles and Practice, 3rd Edn., Addison-Wesley, 2015.

IEC: International Electrotechnical Commission: Multimedia systems and equipment, Colour measurement and management Part 2-1: Colour management, Default RGB colour space, sRGB, IEC 61966-2-1:1999, iCS codes: 33.160.60, 37.080 - TC 100, 1999.

Jakob, W. and Marschner, S.: Manifold exploration: a Markov Chain Monte Carlo technique for rendering scenes with difficult specular transport, ACM Trans. Graph. (TOG) - Proc. of SIGGRAPH 2012, 31, 1-13, doi:10.1145/2185520.2185554, 2012.

Jensen, H. W.: Global Illumination Using Photon Maps, Proc. of the Eurographics Workshop on Rendering Techniques '96, 2130, 1996.
Jähne, B.: EMVA 1288 Standard for Machine Vision, Optik \& Photonik, 5, 53-54, doi:10.1002/opph.201190082, 2010.

Kajiya, J. T.: The rendering equation, ACM SIGGRAPH Computer Graphics, 20, 143-150, doi:10.1145/15886.15902, 1986.

Kaplanyan, A. S., Hanika, J., and Dachsbacher, C.: The NaturalConstraint Representation of the Path Space for Efficient Light Transport Simulation, ACM Trans. Graph. (TOG) - Proc. of SIGGRAPH 2014), 33, 1-13, doi:10.1145/2601097.2601108, 2014.

Kelemen, C., Szirmay-Kalos, L., Antal, G., and Csonka, F.: A Simple and Robust Mutation Strategy for the Metropolis Light Transport Algorithm, Computer Graphics Forum (Proc. of Eurographics), 21, 531-540, doi:10.1111/1467-8659.t01-1-00703, 2002.

Keller, A.: Instant Radiosity, ACM Proc. of SIGGRAPH '97, 4956, doi:10.1145/258734.258769, 1997.

Kettunen, M., Manzi, M., Aittala, M., Lehtinen, J., Durand, F., and Zwicker, M.: Gradient-domain path tracing, ACM Trans. Graph. (TOG) - Proc. of SIGGRAPH 2012, 34, 1-13, doi:10.1145/2766997, 2015.

Korndörfer, J.: Interaktive Zerlegung von Festkörpern in Bruchstücke, diploma thesis, Universität Karlsruhe (TH), 2011.

Lafortune, E. P. and Willems, Y. D.: Bi-Directional Path Tracing, Proc. of Compugraphics '93, 145-153, 1993.

Martinet, A., Galin, E., Desbenoit, B., and Hakkouche, S.: Procedural modeling of cracks and fractures, Proc. of the Shape Modelling International (Short Paper), 346-349, doi:10.1109/SMI.2004.1314524, 2004.

Nicodemus, F. E., Richmond, J. C., Hsia, J. J., Ginsberg, I. W., and Limperis, T.: Geometric Considerations and Nomenclature for Reflectance, Monograph 161, National Bureau of Standards (US), Washington, D.C., USA, 1977.

Popov, S., Ramamoorthi, R., Durand, F., and Drettakis, G.: Probabilistic Connections for Bidirectional Path Tracing, Proc. of the 26st Eurographics Symposium on Rendering (EGSR'15), doi:10.1111/cgf.12680, 2015.

Retzlaff, M.-G., Stabenow, J., and Dachsbacher, C.: Synthetic image acquisition and procedural modeling for automated optical inspection (AOI) systems, Forum Bildverarbeitung 2014, 47-59, doi:10.5445/KSP/1000043608, 2014

Retzlaff, M.-G., Stabenow, J., Beyerer, J., and Dachsbacher, C.: Synthesizing images using parameterized models for automated optical inspection (AOI), tm - Technisches Messen, 82, 251-261, doi:10.1515/teme-2014-0041, 2015.

Retzlaff, M.-G., Richter, M., Längle, T., Beyerer, J., and Dachsbacher, C.: Combining synthetic image acquisition and machine learning: Accelerated design and deployment of sorting systems, Forum Bildverarbeitung 2016, 49-61, 2016.

Schrade, E., Hanika, J., and Dachsbacher, C.: Sparse high-degree polynomials for wide-angle lenses, Computer Graphics Forum (Proc. Eurographics Symposium on Rendering), 35, 89-97, 2016.

Segal, M. and Akeley, K.: The OpenGL Graphics System: A Specification (Ver. 4.2 (Core Profile) - 27 April 2012), Khronos Group, 2012.

Smith, T. and Guild, J.: The C.I.E. Colorimetric Standards and Their Use, Transactions of the Optical Society, 33, 73-134, doi:10.1088/1475-4878/33/3/301, 1931-1932. 
Veach, E.: Robust Monte Carlo Methods for Light Transport Simulation, Ph.d. dissertation, Stanford University, 1998.

Venable, Jr., W. H. and Hsia, J. J.: Describing Spectrophotometric Measurements, Technical Note 594-9, National Bureau of Standards (US), Washington, D.C., USA, 1974.
Yang, J. C., Hensley, J., Grün, H., and Thibieroz, N.: Real-Time Concurrent Linked List construction on the GPU, Proc. of the 21st Eurographics Symposium on Rendering (EGSR'10), 1297 1304, doi:10.1111/j.1467-8659.2010.01725.x, 2010. 